\title{
Recurring Lower Abdominal Pain and Fever as Initial Presentation of Adult Onset Still's Disease in the Absence of Arthralgia and Other System Involvement
}

\author{
Abuajela Sreh, Nithyananda Rajaiah, Mohammad Saim \\ Acute Internal Medicine Department, Walsall Manor Hospital, Walsall, UK
}

Received: $11 / 04 / 2017$

Accepted: 27/04/2017

Published: 09/05/2017

How to cite this article: Sreh A, Rajaiah N, Saim M. Recurring lower abdominal pain and fever as initial presentation of adult onset Still's disease in the absence of athralgia and other system involvement. EJCRIM 2017;4: doi:10.12890/2017_000619

Conflicts of Interests: The Authors declare that there are no competing interests.

Acknowledgements: The Authors would like to thank Liz Askew, Evidence based information specialist librarian, Walsall Manor Hospital, Walsall, United Kingdom. Dr. Sreh and Dr. Rajaiah would like to state that they contributed equally to the manuscript.

This article is licensed under a Commons Attribution Non-Commercial 4.0 License

\section{ABSTRACT}

A 34 year-old Afro-Caribbean female presented with recurring episodes of fever and lower abdominal pain over a period of two months not improving despite courses of antibiotics for possible recurrent urinary tract infections. On admission to hospital, patient was treated for a possible pyelonephritis or pelvic inflammatory disease (PID). Extensive investigations into possible source of infection were carried out. However, all of the repeated microbiological cultures were normal. Patient was investigated further for other possible causes including connective tissue disease, haematological disorders, or neoplasm, all of which were normal. Diagnosis of adult onset Still's disease (AOSD) was confirmed by a rheumatologist based on Yamaguchi's diagnostic criteria for AOSD alongside significantly raised serum ferritin. Patient was treated with steroids to which she showed remarkable clinical improvement alongside marked reduction in her serum ferritin levels.

\section{LEARNING POINTS}

- The four giant causes of fever of unknown origin are: infections, connective tissue diseases, haematological disorders, and malignancies.

- AOSD is a diagnosis of exclusion using Yamaguchi criteria alongside raised ferritin levels (the most sensitive biomarker).

- Most AOSD patients will respond to steroid treatment. However, approximately one third of the patients require further immunosuppression to achieve disease remission.

\section{KEYWORDS}

Adult onset Still's disease, AOSD, fever of unknown origin, pyrexia, lower abdominal pain

\section{INTRODUCTION}

AOSD is a rare multi-system inflammatory disorder. The typical clinical triad of AOSD is cyclical high grade fever, arthralgia, and rash ${ }^{[1]}$. All cases reported in the literature presented with a minimum of fever and arthralgia/arthritis alongside other variable systematic effects ${ }^{[2]}$. The case presented in this article depicts the challenge of making a confirmed diagnosis of AOSD in a patient initially presenting atypically with mild lower abdominal pain and fever of unknown origin in the absence of arthralgia or joint involvement. The diagnosis of AOSD was reached after conducting a series of investigations that ruled out any infectious, autoimmune, haematological, or neoplastic causes of pyrexia of unknown origin. Diagnosis was made on the basis of the clinically-approved Yamaguchi criteria for the diagnosis of AOSD alongside significantly raised serum ferritin ${ }^{[3]}$. 


\section{CASE DESCRIPTION}

A 34 year-old Afro-Caribbean presented at the emergency department with a 2 month history of fever and mild lower abdominal pain. She was initially treated in the community for recurrent urinary tract infections but her symptoms persisted so she self-presented to the emergency department. Patient reported gradual onset of fever and intermittent lower abdominal pain. Patient also recently developed a sore throat for which she was treated in the community for upper respiratory tract infection. Patient denied any genitourinary tract symptoms. Patient neither had any changes in her bowel habit throughout her illness period nor did she experience any per rectum bleeding. Patient had no respiratory or neurological symptom and denied any rash, weight loss, night sweats, arthralgia, or lumps or bumps anywhere in her body. Patient is married and has one child. Last menstrual period was one week prior to her admission. Patient had been to France with her family 3 weeks prior to her presentation. She declined any illness or exposure to ill people while abroad. There was no other recent travel history. She was otherwise medically fit and takes no regular medications. She denied taking or injecting any illicit drugs. There was no family history of recent illness, connective tissue, haematological diseases, or malignancies.

Patient was alert on clinical examination with no pallor, jaundice, cyanosis, or clubbing. Vital signs on admission showed temperature of 39.4 degrees and tachycardia at 126 beats per minute, otherwise normal respiratory rate, blood pressure, and oxygen saturation of $99 \%$ on air. Patient was noted to have enlarged cervical lymph nodes but normal palpation of other lymph node groups. Cardio-respiratory examinations were unremarkable and abdomen was soft with mild suprapubic tenderness in the absence of any organ enlargement, guarding, or rebound tenderness with normal bowel sounds on auscultation. No focal neurology or any signs of meningism (Fig. 1).

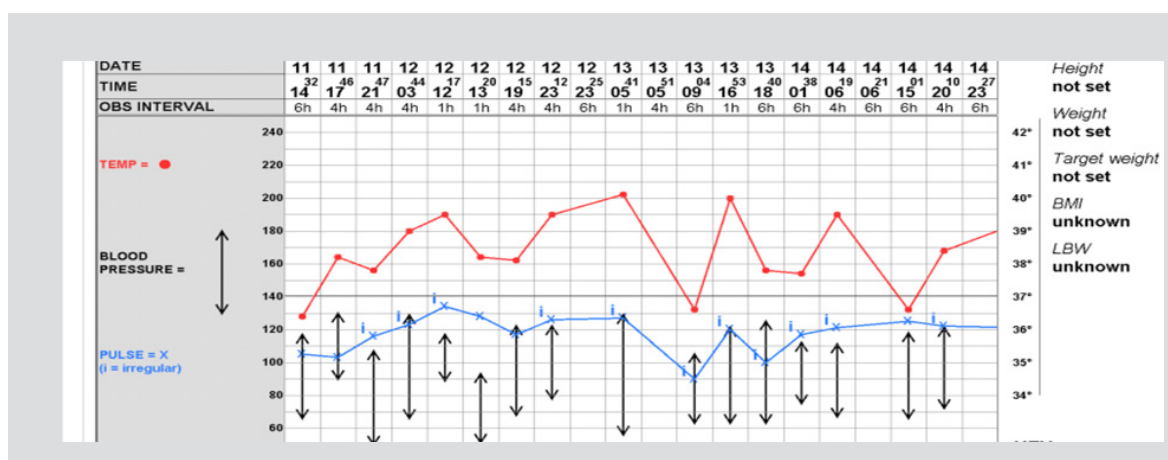

Figure 1. Cyclical pattern of patient high grade fever alongside the other vital signs through the first five days of patient admission.

Methods and procedures

Initial investigations revealed normal Chest X-ray and normal urine analysis. Routine bloods showed moderately raised inflammatory markers with mildly deranged liver function test. Patient was initially treated for suspected genitourinary sepsis (possible pyelonephritis or PID) by intravenous fluids and antibiotics. Patient was then reviewed by the gynaecology team (on basis of a suspected diagnosis of PID as well as a positive beta human chorionic gonadatropin (beta-HCG). Trans-vaginal ultrasound scan was performed urgently and showed normal examination with no products of conception ruling out pregnancy or any other gynaecological disease.

Patient continued to spike temperature despite using different IV broad spectrum antibiotics for pyrexia of unknown origin as per the recommendations of the local microbiologist.

All microbiological investigations were normal including respiratory, urinary, and stool cultures as well as gynaecological swab cultures. Full bacterial and viral screen for persistent fever were normal (including HIV and hepatitis). Patient also had normal Sickle cell and haemoglobinopathy screen, immunological screen, and rheumatoid arthritis screen. Patient was noted to have positive anti-nucleic acid (ANA) antibodies with normal lupus screen, ANCA and ENA antibodies. Trans-thoracic echo scan was performed which ruled out any vegetation with normal heart structures. Computed tomography (CT) of the chest, abdomen, and pelvis was performed which showed no focus of infection or malignancy explaining the persistent fever. A haematologist reviewed the patient and performed a bone biopsy, which showed normal bone marrow cells in the absence of malignant cells.

Patient found to have significantly raised serum ferritin $(47869 \mu \mathrm{g} / \mathrm{L})$ with raised erythrocyte sedimentation rate (ESR) as well as lactate dehydrogenase (LDH 2000 units/litres). Patient symptoms, signs, and investigations scored only one out of eleven (for positive ANA) on the American college of rheumatology criteria for systemic lupus erythromatosis (SLE), excluding a diagnosis of SLE. Patient neither exhibited changes in her bowel habit throughout her illness period nor did she notice any blood or mucous in her faeces. Patient neither showed symptoms suggesting intestinal malabsorption nor did she have any extra-intestinal features that could point to a diagnosis of inflammatory 
bowel disease (IBD). The lack of the cardinal clinical features of IBD alongside the significantly raised ferritin with normal CT abdomen and pelvis made a diagnosis of IBD very unlikely ${ }^{[4-6]}$.

Patient was stable and was discharged on regular paracetamol and ibuprofen. Initial diagnosis was pyrexia of unknown origin after all of the repeatedly normal microbiological and immunological investigations alongside full body CT scan and bone biopsy. Patient was reviewed in outpatient clinic one week post discharge where she was readmitted again with persistent fever. Full microbiological work-up was repeated and was normal. A diagnosis of exclusion of AOSD was reached and confirmed by a rheumatologist using Yamaguchi diagnostic criteria alongside the significantly raised serum ferritin as well ESR and $\mathrm{LDH}^{[3]}$. Patient satisfied two major criteria (fever of more than $39^{\circ} \mathrm{Celsius}$ lasting more than a week alongside leucocytosis with more than $80 \%$ polymorphoneuclear cells) in addition to 3 minor criteria (sore throat, lymphadenopathy, and deranged liver function). All the antibiotics were stopped and patient was started on high-dose prednisolone, to which patient responded remarkably well and so steroids were tapered over four weeks. Patient improved significantly when she was reviewed in the rheumatology outpatient clinic. Patient was explained the possible need for disease modifying anti-rheumatic drugs (DMARDs) if her symptoms relapse again in the future.

\section{DISCUSSION}

Adult onset Still's disease (AOSD) is a rare multisystem inflammatory disease first described by Bywaters in 1971 after studying 17 patients presenting with similar features to Still's disease in children ${ }^{[7,8]}$. AOSD tends to affect young adults (most commonly 16-35 years olds) and has an annual incidence rate of less than 1 in 100,000. It affects both genders equally ${ }^{[8,9]}$.

There is no known cause but it is believed that its aetiology is multifactorial with a combination of genetic and environmental (mainly infectious) factors having been identified ${ }^{[10]}$. AOSD is still a diagnostic challenge to most physicians and this diagnosis has been reached by exclusion in most reported cases. The most commonly used diagnostic criteria to help establish a diagnosis of AOSD was developed by Yamaguchi et al. ${ }^{[3]}$. For an AOSD diagnosis to be confirmed against Yamaguchi's diagnostic criteria, the patient must have a minimum of two major criteria and three minor criteria.

A major systematic review of AOSD by Ohta et al. revealed that all of their 228 reported AOSD cases presented with arthralgia except for one particular case ${ }^{[11]}$. This case was originally reported by Bywaters in 1971 but no details of the associated symptoms and signs were provided $^{[8]}$. In addition, $13 \%$ of the 228 cases reported by Ohta et al presented with abdominal pain without clear details on whether or not any case had abdominal pain associated with joint involvement in the initial presentation ${ }^{[11]}$.

Treatment of AOSD usually starts with non-steroidal anti-inflammatory drugs (NSAIDs) with around $80 \%$ of patients requiring steroids to achieve full recovery. Approximately $35 \%$ of patients require DMARDs such as methotrexate, azathioprine, cyclosporine, and cylclophosphamide as maintenance therapy to combat the disease ${ }^{[13]}$. There is good evidence supporting the use of biological agents (mainly anti-TNF agents such us infliximab and etanercept) especially in refractory AOSD patients ${ }^{[13-15]}$.

Most patients diagnosed with AOSD recovered well and their function returned to normal. However, a consistent finding in the literature is that approximately one third of ASOD patients develop erosive Rheumatoid pattern polyarthritis ${ }^{[11-15]}$. This emphasises the importance of considering early AOSD diagnosis in any patient admitted with pyrexia of unknown origin with normal microbiological, autoimmune screen, body imaging, and bone marrow biopsy.

\section{REFERENCES}

1. Mihaescu R, Serban C, Mozos I, Sirbu E. Adult-onset still's disease presenting as persistent fever of unknown origin: Case report and review of literature. J Banagladesh Coll Phys Surg 2014; 32: 224-228.

2. Carreño MA, Carvallo A, Trejo C, Ballestero F, Martinez C. Adult Still's disease. A great simulator: retrospective review of 20 patients. Rev Med Chil 2009;137:1010-1016.

3. Yamaguchi M, Ohta A, Tsunematsu T, Kasukawa R et al. Preliminary criteria for classification of adult Still's disease. J Rheumatol 1992;19:424-430.

4. Ha F, Khalil H. Crohn's disease: a clinical update. Therap Adv Gastroenterol 2015;8:352-9. doi: 10.1177/1756283X15592585.

5. Crohn's disease; NICE CKS, April 2015 (UK access only). Available from URL: https://cks.nice.org.uk/crohns-disease

6. Gasche C, Lomer MCE, Cavill I, Weiss G. Iron, anaemia, and inflammatory bowel diseases. Gut 2004;53:1190-1197.

7. Magadur-Joly G, Billaud E, Barrier JH, Pennec YL et al. Epidemiology of adult Still's disease: estimate of the incidence by a retrospective study in west France. Ann Rheum Dis 1995;54:587-90.

8. Bywaters E. Still's disease in the adults. Ann Rheum Dis 1971;30:121-133.

9. Dunne CA, Davies J. Epidemiology of adult Still's disease. Ann Rheum Dis 1996;55:272.

10. Shin SH, Shin DH, Ryu PY, Chung SS, Rhee JH. Proinflammatory cytokine profile in Vibrio vulnificus septicemic patients' sera. FEMS Immunol Med Microbiol 2002;33:133-138.

11. Ohta A, Yamaguchi M, Kaneoka H, Nagayoshi T, Hiida M. Adult Still's disease: review of 228 cases from the literature. J Rheumatol 1987;14:1139-46.

12. Akintayo RO, Adelowo O. Adult-onset Still's disease in a Nigerian woman. BMJ Case Rep 2015. doi:10.1136/bcr-2015-21078.

13. Manger B, Rech J, Schett G. Use of methotrexate in adult-onset Still's disease. Clin Exp Rheumatol 2010;28 (Suppl. 61):S168-S171.

14. Kokkinos A, Iliopoulos A, Greka P, Efthymiou A, et al. Successful treatment of refractory adult-onset still's disease with infliximab. A prospective, non-comparative series of four patients. Clin Rheumatol 2004;23:45-49.

15. Husni ME, Maier AL, Mease PJ, Overman SS et al. Etanercept in the treatment of adult patients with Still's disease. Arthritis Rheum 2002;46:1171-1176. 